\title{
Thirty- and ninety-day outcomes after sublobar resection with and without brachytherapy for non-small cell lung cancer: Results from a multicenter phase III study
}

\author{
Hiran C. Fernando, MD, ${ }^{a}$ Rodney J. Landreneau, MD, ${ }^{\mathrm{b}}$ Sumithra J. Mandrekar, PhD, ${ }^{\mathrm{c}}$ \\ Shauna L. Hillman, MS, ${ }^{\mathrm{c}}$ Francis C. Nichols, MD, ${ }^{\mathrm{c}}$ Bryan Meyers, MD, ${ }^{\mathrm{d}}$ Thomas A. DiPetrillo, MD, \\ Dwight E. Heron, MD, ${ }^{\mathrm{b}}$ David R. Jones, MD, ${ }^{\mathrm{f}}$ Benedict D. T. Daly, MD, ${ }^{\mathrm{a}}$ Sandra L. Starnes, MD, ${ }^{\mathrm{g}}$ \\ Angelina Tan, BS, ${ }^{\mathrm{c}}$ and Joe B. Putnam, $\mathrm{MD}^{\mathrm{h}}$
}

\begin{abstract}
Objective: Sublobar resection (SR) is commonly used for patients considered high risk for lobectomy. Nonoperative therapies are increasingly being reported for patients with similar risk because of perceived lower morbidity. We report 30- and 90-day adverse events (AEs) from American College of Surgeons Oncology Group Z4032, a multicenter phase III study for high-risk patients with stage I non-small cell lung cancer.
\end{abstract}

Methods: Data from 222 evaluable patients randomized to SR $(n=114)$ or SR with brachytherapy $(n=108)$ are reported. AEs were recorded using the Common Terminology Criteria for Adverse Events, Version 3.0, at 30 and 90 days after surgery. Risk factors (age, percent baseline carbon monoxide diffusion in the lung [DLCO\%], percent forced expiratory volume in 1 second [FEV1\%], upper lobe vs lower lobe resections, performance status, surgery approach, video-assisted thoracic surgery vs open and extent, and wedge vs segmentectomy) were analyzed using a multivariable logistic model for their impact on the incidence of grade 3 or higher (G3+) AEs. Respiratory AEs were also specifically analyzed.

Results: Median age, FEV1\%, and DLCO \% were similar in the 2 treatment groups. There was no difference in the location of resection (upper vs lower lobe) or the use of segmental or wedge resections. There were no differences between the groups with respect to "respiratory" G3+AEs (30 days: $14.9 \%$ vs $19.4 \%, P=.35 ; 0-90$ days: $19.3 \%$ vs $25 \%, P=.31$ ) and "any" G3+ AEs (30 days: $25.4 \%$ vs $30.6 \%, P=.37 ; 0-90$ days: $29.8 \%$ vs $37 \%, P=.25$ ). Further analysis combined the 2 groups. Mortality occurred in 3 patients $(1.4 \%)$ by 30 days and in 6 patients $(2.7 \%)$ by 90 days. Four of the 6 deaths were thought to be due to surgery. When considered as continuous variables, FEV $1 \%$ was associated with "any" G3+ AE at days 0 to $30(P=.03$; odds ratio $[\mathrm{OR}]=0.98)$ and days 0 to 90 $(P=.05 ; \mathrm{OR}=0.98)$, and $\mathrm{DLCO} \%$ was associated with "respiratory" $\mathrm{G} 3+\mathrm{AE}$ at days 0 to $30(P=.03$; $\mathrm{OR}=0.97)$ and days 0 to $90(P=.05 ; \mathrm{OR}=0.98)$. Segmental resection was associated with a higher incidence of any G3+ AE compared with wedge resection at days 0 to $30(40.3 \%$ vs $22.7 \%$; OR $=2.56 ; P<.01)$ and days 0 to $90(41.5 \%$ vs $29.7 \%$; OR $=1.96 ; P=.04)$. The median FEV $1 \%$ was $50 \%$, and the median DLCO $\%$ was $46 \%$. By using these median values as potential cutpoints, only a DLCO $\%$ of less than $46 \%$ was significantly associated with an increased risk of "respiratory" and "any" G3+AE for days 0 to 30 and 0 to 90 .

Conclusions: In a multicenter setting, SR with brachytherapy was not associated with increased morbidity compared with SR alone. SR/SR with brachytherapy can be performed safely in high-risk patients with non-small cell lung cancer with low 30- and 90-day mortality and acceptable morbidity. Segmental resection was associated with increased "any" G3+AE, and DLCO $\%$ less than $46 \%$ was associated with "any" G3+AE and "respiratory" G3+ AE at both 30 and 90 days. (J Thorac Cardiovasc Surg 2011;142:1143-51)

Earn CME credits at

http://cme.ctsnetjournals.org
From the Boston Medical Center, ${ }^{\mathrm{a}}$ Boston, Mass; University of Pittsburgh, ${ }^{\mathrm{b}}$ Pittsburgh, Pa; Mayo Clinic, ${ }^{\mathrm{c}}$ Rochester, Minn; Washington University of St Louis, ${ }^{\mathrm{d}}$ St Louis, Mo; Rhode Island Hospital, ${ }^{e}$ Providence, RI; University of Virginia, ${ }^{f}$ Charlottesville, Va; University of Cincinnati, ${ }^{\mathrm{g}}$ Ohio; and Vanderbilt University Medical Center, ${ }^{\mathrm{h}}$ Nashville, Tenn.

Supported by NCI U10 grant CA076001 and by an additional grant from Oncura, Princeton, New Jersey.

Disclosures: Authors have nothing to disclose with regard to commercial support. Read at the 91st Annual Meeting of The American Association for Thoracic Surgery, Philadelphia, Pennsylvania, May 7-11, 2011.
Sublobar resection (SR) is usually reserved for patients with non-small cell lung cancer (NSCLC) who are considered high risk for lobectomy. The principal reason for selective use in higher-risk patients is the higher locoregional

Received for publication May 8, 2011; revisions received July 9, 2011; accepted for publication July 25, 2011; available ahead of print Aug 29, 2011.

Address for reprints: Hiran C. Fernando, MD, Department of Cardiothoracic Surgery, Boston Medical Center, 88 East Newton Street, Boston, MA 02118 (E-mail: Hiran. Fernando@bmc.org).

0022-5223/\$36.00

Copyright (C) 2011 by The American Association for Thoracic Surgery doi:10.1016/j.jtcvs.2011.07.051 


$$
\begin{aligned}
& \text { Abbreviations and Acronyms } \\
& \text { ACOSOG }=\text { American College of Surgeons } \\
& \text { Oncology Group } \\
& \mathrm{AE} \quad=\text { adverse event } \\
& \text { ASA }=\text { American Society of } \\
& \text { Anesthesiologists } \\
& \mathrm{CI}=\text { confidence interval } \\
& \text { CTC }=\text { Common Terminology Criteria } \\
& \text { DLCO }=\text { carbon monoxide diffusion in the } \\
& \text { lung } \\
& \mathrm{FEV}_{1}=\text { forced expiratory volume in } 1 \text { second } \\
& \text { NSCLC }=\text { non-small cell lung cancer } \\
& \mathrm{OR}=\text { odds ratio } \\
& \text { SBRT = stereotactic body radiation therapy } \\
& \text { SR }=\text { sublobar resection } \\
& \text { SRB = sublobar resection with } \\
& \text { intraoperative brachytherapy }
\end{aligned}
$$

recurrence rate after SR compared with lobectomy. ${ }^{1}$ One approach that may reduce the incidence of local recurrence is the addition of adjuvant brachytherapy. ${ }^{2-4} \mathrm{Z} 4032$ is a prospective randomized clinical trial by the American College of Surgeons Oncology Group (ACOSOG) that compares SR with intraoperative brachytherapy (SRB) with SR alone. This study has recently completed accrual. The primary outcome of interest of the study is 2-year local control, and this end point will be reported when sufficient follow-up becomes available. The current report examines the incidence and severity of adverse events (AEs) occurring at both 30 and 90 days after surgery from this multicenter randomized prospective study. This is of particular importance because nonoperative therapies, such as stereotactic body radiation therapy (SBRT) and radiofrequency ablation, are gaining increasing attention within the medical community, even for patients who are candidates for operation. ${ }^{4,5}$ The toxicity profiles of the various lung cancer therapies are important considerations when discussing treatment options with patients. We report the incidence and severity of AEs after SR in the high-risk population selected for Z4032.

\section{MATERIALS AND METHODS}

Eligible patients for this study included patients with stage I lung cancers $3 \mathrm{~cm}$ or less in maximum diameter (ie, stage IA or the subset of stage IB with visceral pleural involvement) on preoperative computed tomography scan. Patients were defined as high risk for lobectomy if they met at least 1 major criterion or 2 minor criteria as described in Table 1 . In addition to meeting these criteria, patients had to be evaluated by an ACOSOG-approved thoracic surgeon and considered not to be a candidate for lobectomy or to be too high risk for any form of pulmonary resection. Patients considered medically inoperable (but who met these criteria) were usually referred for nonoperative therapies, such as radiofrequency ablation or SBRT. We did not record the details of screened patients who met the major and minor criteria described above who were not offered participation in this study. To confirm that patients did not have nodal involvement, all suspicious lymph nodes seen on positron emission tomography or computed tomography scan required biopsy by mediastinoscopy, endobronchial ultrasound, or sampling at the time of resection. SR included wedge or segmental resection and could be performed by videoassisted thoracic surgery or thoracotomy. Two methods of brachytherapy were allowed. ${ }^{6,7}$ The method used was at the discretion of the treating surgeon. In the first technique, polyglactin sutures containing ${ }^{125}$ I seeds (Oncura, Inc, Princeton, NJ) were placed parallel to and $5 \mathrm{~mm}$ away from the staple line on each side of the resection margin. The suture strands were fixed to the lung surface with several 3.0 silk or polyglactin sutures placed 1 to $2 \mathrm{~cm}$ apart. With the second brachytherapy technique, a polyglycolic mesh implant was created during the procedure. The same ${ }^{125} \mathrm{I}$ suture strands were woven into a piece of Vicryl mesh. The strands were placed at $1-\mathrm{cm}$ intervals. The mesh was then sutured over the staple line. The dosimetry goal of the brachytherapy was to deliver $100 \mathrm{~Gy}$ at 5 to $7 \mathrm{~mm}$ along the central axis of the resection margin.

AEs were recorded using the Common Terminology Criteria (CTC) for Adverse Events Version 3.0. ${ }^{8}$ The CTC is a broad classification of AE with several defined categories. Within each category, AEs are listed and accompanied by a description of severity (grade). Grade 1 is mild, grade 2 is moderate, grade 3 is severe, grade 4 is life-threatening or disabling $\mathrm{AE}$, and grade 5 is death related to the $\mathrm{AE}$.

AEs were analyzed at 0 to 30 days and again at 0 to 90 days. For the purpose of this report, we limit discussion to grade 3 and higher (3+) AE. Because this group was considered high risk primarily on the basis of lung function, 2 groups of AE were studied: "any AE" or "respiratory $\mathrm{AE}$ ", where "respiratory $\mathrm{AE}$ " included adult respiratory distress syndrome, aspiration, bronchospasm, bronchostenosis, dyspnea, hypoxia, pleural effusion, pneumonitis, chest tube drainage or leak, prolonged intubation, pulmonary-other, and pneumonia as defined by the CTC.

All patients provided written informed consent before trial enrollment in accordance with applicable guidelines. At each participating site, institutional review board approval was obtained in accord with an assurance filed with and approved by the US Department of Health and Human Services.

\section{Statistical Analysis}

Chi-square tests for categoric variables and Wilcoxon rank-sum tests for continuous variables were used to compare the baseline patient characteristics between the SR and the SRB arms. We compared the 2 treatment arms for the incidence of any grade $3+\mathrm{AE}$ and any grade $3+$ respiratory AE using a Fisher exact test. Risk factors for AEs (age, baseline carbon monoxide diffusion in the lung [DLCO]\%, and forced expiratory volume in 1 second $\left[\mathrm{FEV}_{1}\right] \%$ considered as continuous variables, upper lobe vs lower lobe resection, and performance status) were analyzed using a multivariable logistic model for any grade $3+$ and grade $3+$ respiratory AEs at 0 to 30 days and 0 to 90 days. In addition to these factors, surgery extent (wedge vs segmentectomy) and type (thoracotomy vs video-assisted thoracic surgery) were also considered for any grade $3+\mathrm{AEs}$ outcomes at 0 to 30 and 0 to 90 days. Odds ratios (ORs) and $95 \%$ confidence intervals (CIs) are reported, where the OR estimates for a continuous covariate correspond to a 1-unit increase. Optimal cutpoints were therefore explored to define the high-risk versus low-risk categories for the baseline $\mathrm{DLCO} \%$ and $\mathrm{FEV}_{1} \%$ using datadependent methods (mean or median) and outcome-based approaches (graphic diagnostic plots and the minimum $P$ value approach). ${ }^{9}$ Subsequently, univariable logistic regression models using the categorized $\mathrm{DLCO} \%$ and $\mathrm{FEV}_{1} \%$ were explored. In addition, the entry criteria used to define the high-risk subset were explored further by first analyzing patients eligible by at least 2 minor criteria and by including 
TABLE 1. Major and minor eligibility criteria for Z4032 trial*

\begin{tabular}{|c|c|c|}
\hline & $\mathbf{S R} \dagger(\mathbf{N}=\mathbf{1 1 4})$ & $\mathbf{S R B} \dagger(\mathbf{N}=\mathbf{1 0 8})$ \\
\hline \multicolumn{3}{|l|}{ Major criteria } \\
\hline 1. $\mathrm{FEV}_{1} \leq 50 \%$ predicted & $67(58.8 \%)$ & $49(45.4 \%)$ \\
\hline 2. DLCO $\leq 50 \%$ predicted & $72(63.2 \%)$ & $74(68.5 \%)$ \\
\hline \multicolumn{3}{|l|}{ Minor criteria } \\
\hline 1. Age $\geq 75 \mathrm{y}$ & $43(37.7 \%)$ & $42(38.9 \%)$ \\
\hline 2. $\mathrm{FEV}_{1} 51 \%-60 \%$ predicted & $18(15.8 \%)$ & $25(23.1 \%)$ \\
\hline 3. DLCO $51 \%-60 \%$ predicted & $19(16.7 \%)$ & $19(17.6 \%)$ \\
\hline $\begin{array}{l}\text { 4. Pulmonary hypertension (defined as pulmonary artery systolic pressure } \\
>40 \mathrm{~mm} \mathrm{Hg} \text { ) as estimated by echocardiography or right heart catheterization }\end{array}$ & $4(3.5 \%)$ & $1(0.9 \%)$ \\
\hline 5. Poor left ventricular function (defined as ejection fraction $\leq 40 \%$ ) & $9(7.9 \%)$ & $3(2.8 \%)$ \\
\hline 6. Resting or exercise arterial $\mathrm{Po}_{2} \leq 55 \mathrm{~mm} \mathrm{Hg}$ or $\mathrm{SPO}_{2} \leq 88 \%$ & $5(4.4 \%)$ & $6(5.6 \%)$ \\
\hline 7. $\mathrm{PCO}_{2}>45 \mathrm{~mm} \mathrm{Hg}$ & $3(2.6 \%)$ & $3(2.8 \%)$ \\
\hline 8. Modified Medical Research Council Dyspnea Scale $\geq 3$ & $31(27.2 \%)$ & $17(15.7 \%)$ \\
\hline
\end{tabular}

Oncology Group-approved thoracic surgeon. $\dagger$ One patient may have multiple criteria.

patients eligible with at least $\mathrm{FEV}_{1} \%$ and $\mathrm{DLCO} \%$ as minor criteria as risk factors in univariable logistic regression models for all the end points considered.

\section{RESULTS}

Z4032 met its target accrual and was permanently closed to enrollment on January 22, 2010. A total of 224 patients were registered. One patient from the SR arm had the intervention at a hospital not approved by the institutional review board hospital and was deemed not evaluable. One patient randomized to the SRB group did not have surgery and was also not evaluable. A total of 222 patients $(\mathrm{SR}=114 ; \mathrm{SRB}=108$ patients $)$ were eligible for analysis (Figure 1).

Table 2 depicts the patient characteristics for the 2 groups. There were no significant differences between the 2 intervention groups in baseline characteristics, except American Society of Anesthesiologists (ASA) class, for which a higher percentage of the SR group was classified as ASA III/IV. The median age, $\mathrm{FEV}_{1} \%$, and DLCO $\%$ were similar for the 2 groups.

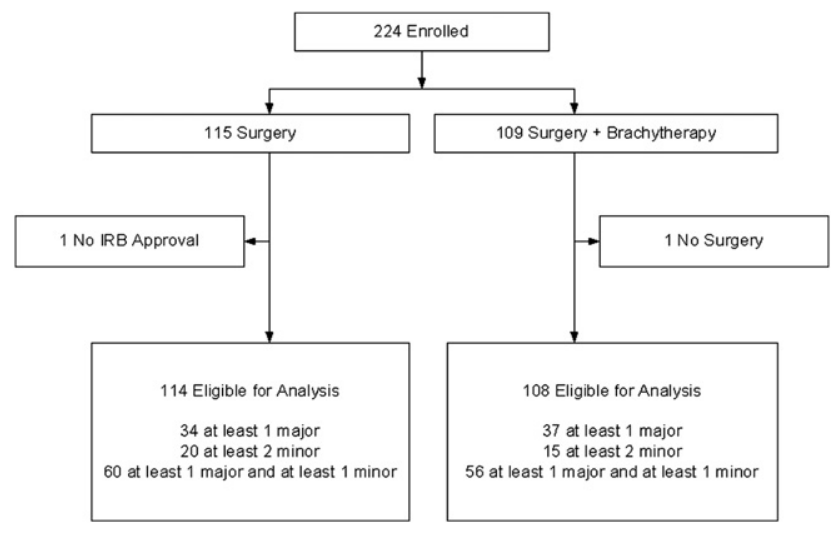

FIGURE 1. Patient consort diagram.
Thirty- and Ninety-Day Mortality: Sublobar Resection Versus Sublobar Resection With Brachytherapy

There were no significant differences between the SR and SRB groups with respect to 30- and 90-day mortality (grade $5 \mathrm{AE})$. Three deaths $(1.4 \%)$ occurred within 30 days. One patient $(0.9 \%)$ in the SR group died of cardiopulmonary arrest. Two patients $(1.9 \%)$ in the SRB group died of cerebrovascular accident and pulmonary embolus. By 90 days, an additional 3 deaths (total, $6 ; 2.7 \%$ ) had occurred. Two patients $(1.8 \%)$ were in the SR group and included cancer progression in the 1 patient found to have $\mathrm{N} 2$ disease on pathologic staging and a fatal cardiac event in 1 patient. One patient $(0.9 \%)$ in the SRB group died of sepsis. Four of the 6 deaths occurring by 90 days were thought to be attributable to the surgery performed.

\section{Thirty- and Ninety-Day Adverse Events: Sublobar Resection Versus Sublobar Resection With Brachytherapy}

Median length of stay was 5 days for each group $(P=.33)$. At 0 to 30 days, grade $3+\mathrm{AE}$ occurred in $29 \mathrm{pa}-$ tients $(25.4 \%)$ in the SR group compared with 33 patients $(30.6 \%)$ in the SRB group $(P=.37)$. Grade $4+$ AEs were also similar, occurring in 8 patients $(7 \%)$ in the SR group compared with 8 patients $(7.4 \%)$ in the SRB group $(P=.90)$. In terms of respiratory $\mathrm{AE}$, grade $3+\mathrm{AE}$ and grade $4+\mathrm{AE}$ occurred in 17 patients $(14.9 \%)$ and 21 patients $(19.4 \%)$ in the SR group $(P=.35)$, and 4 patients $(3.5 \%)$ and 4 patients $(3.7 \%)$ in the SRB group $(P=$ .93 ), respectively. The most common grade $3+$ AEs (defined as occurring in $\geq 5 \%$ patients within each arm) were hemorrhage ( $4.1 \% ; 3$ in the SR group and 6 in the SRB group), dyspnea $(8.6 \% ; 11$ in the SR group and 8 in the SRB group), and hypoxia $(6.3 \% ; 5$ in the SR group and 9 in the SRB group). 
TABLE 2. Baseline patient characteristics

\begin{tabular}{|c|c|c|c|}
\hline & $\begin{array}{c}\text { SR } \\
(\mathrm{N}=\mathbf{1 1 4})\end{array}$ & $\begin{array}{c}\text { SRB } \\
(\mathrm{N}=\mathbf{1 0 8}) \\
\end{array}$ & $P$ value* \\
\hline Age, y & & & $.37 \dagger$ \\
\hline Median (range) & $70(49-85)$ & $72(50-87)$ & \\
\hline Sex & & & .72 \\
\hline Female & $65(57 \%)$ & $59(55 \%)$ & \\
\hline Male & $49(43 \%)$ & $49(45 \%)$ & \\
\hline Ethnicity & & & .16 \\
\hline Hispanic or Latino & $0(0 \%)$ & $2(1.9 \%)$ & \\
\hline Not Hispanic or Latino & $104(91 \%)$ & $91(84 \%)$ & \\
\hline Unknown & $10(9 \%)$ & $15(14 \%)$ & \\
\hline Race & & & .42 \\
\hline White & $107(93.9 \%)$ & $103(95.4 \%)$ & \\
\hline Black & $7(6.1 \%)$ & $4(3.7 \%)$ & \\
\hline Unknown & $0(0 \%)$ & $1(0.9 \%)$ & \\
\hline Baseline performance status & & & .56 \\
\hline 0 & $20(17.5 \%)$ & $25(23.1 \%)$ & \\
\hline 1 & $66(57.9 \%)$ & $60(55.6 \%)$ & \\
\hline 2 & $28(24.6 \%)$ & $23(21.3 \%)$ & \\
\hline T stage & & & .12 \\
\hline $\mathrm{T} 1$ & $114(100 \%)$ & $104(96.3 \%)$ & \\
\hline $\mathrm{T} 2$ & $0(0 \%)$ & $3(2.8 \%)$ & \\
\hline T3 & $0(0 \%)$ & $1(0.9 \%)$ & \\
\hline M stage & & & NA \\
\hline M0 & $114(100 \%)$ & $108(100 \%)$ & \\
\hline $\mathrm{N}$ stage & & & .37 \\
\hline No & $113(99.1 \%)$ & $107(99.1 \%)$ & \\
\hline N1 & $0(0 \%)$ & $1(0.9 \%)$ & \\
\hline $\mathrm{N} 2$ & $1(0.9 \%)$ & $0(0 \%)$ & \\
\hline Surgery in upper lobe & & & .07 \\
\hline No & $38(33.3 \%)$ & $49(45.4 \%)$ & \\
\hline Yes & $76(66.7 \%)$ & $59(54.6 \%)$ & \\
\hline \multicolumn{4}{|l|}{ Surgery type } \\
\hline VATS & $79(69.3 \%)$ & $65(60.2 \%)$ & .16 \\
\hline Thoracotomy & $35(30.7 \%)$ & $43(39.8 \%)$ & \\
\hline Surgery extent & & & .29 \\
\hline Segmentectomy & $38(33.3 \%)$ & $29(26.9 \%)$ & \\
\hline Wedge resection & $76(66.7 \%)$ & $79(73.1 \%)$ & \\
\hline $\begin{array}{c}\text { ASA class (III/IV vs I/II) } \\
\text { on surgery day } \ddagger\end{array}$ & & & .02 \\
\hline $\mathrm{I} / \mathrm{II}$ & $10(8.8 \%)$ & $21(19.8 \%)$ & \\
\hline III/IV & $104(91.2 \%)$ & $85(80.2 \%)$ & \\
\hline Baseline DLCO \% & & & $.36 \dagger$ \\
\hline $\mathrm{N}$ & 111 & 106 & \\
\hline Median (range) & $47(18-97)$ & $44(8-137)$ & \\
\hline Baseline $\mathrm{FEV}_{1} \%$ & & & $.25 \dagger$ \\
\hline $\mathrm{N}$ & 114 & 107 & \\
\hline Median (range) & $48(22-117)$ & $53(25-110)$ & \\
\hline
\end{tabular}

VATS, Video-assisted thoracic surgery; NA, not available. *Chi-square test. $\dagger$ Wilcoxon rank-sum test. $\ddagger$ Excludes 2 SRBs with missing data.

At 0 to 90 days, grade $3+\mathrm{AE}$ occurred in 34 patients $(29.8 \%)$ in the SR group and 40 patients $(37 \%)$ in the SRB group $(P=.25)$. Grade $4+$ events were also similar $(P=.71)$, occurring in 10 patients $(8.8 \%)$ in the SR group and 8 patients $(7.4 \%)$ in the SRB group. In terms of respiratory $\mathrm{AE}$, grade $3+\mathrm{AE}$ and grade $4+\mathrm{AE}$ occurred in 22 patients $(19.3 \%)$ and 27 patients $(25 \%)$, and 6 patients $(5.3 \%)$ and 4 patients $(3.7 \%)$ in the SR and SRB groups, respectively $(P=.31 ; P=.58)$. The most common grade $3+$ AEs (defined as occurring in $\geq 5 \%$ patients within each arm) were the same as those reported for 0 to 30 days, with an exception of increasing reports of dyspnea occurring in 29 patients $(13.1 \%, 14$ in the SR group and 15 in the SRB group).

Twelve patients (9 in the SR group and 3 in the SRB group) had poor left ventricular function at study entry. There was no association between ASA class and poor left ventricular function (Fisher exact $P=1.0$ ). Logistic regression analysis considering ASA class and treatment arm as predictors of grade $3+$ cardiovascular AEs showed no significant effect of ASA class (days 0-30: $P=.92$; OR, 0.92; 95\% CI, 0.19-4.45; days 0-90: $P=.94$; OR, 1.06 ; $95 \% \mathrm{CI}$, $0.22-5.02)$.

\section{Thirty- and Ninety-Day Adverse Events: All Patients}

The 2 groups were therefore combined for further analyses. We found that patients based on "any 2 minor criteria" and "at least $\mathrm{FEV}_{1} \%$ and DLCO $\%$ as minor criteria" were not significant predictors of AE outcomes. In a multivariable model of $\mathrm{FEV}_{1} \%$ (considered as a continuous variable) without $\mathrm{DLCO} \%, \mathrm{FEV}_{1} \%$ was associated with any grade $3+\mathrm{AE}$ at days 0 to $30(P=.04$; OR, $0.98 ; 95 \% \mathrm{CI}$, $0.97-1.00)$ and respiratory grade $3+\mathrm{AE}$ at days 0 to 30 $(P=.04$; OR, $0.98 ; 95 \% \mathrm{CI}, 0.96-1.00)$ and days 0 to 90 $(P=.03$; OR, 0.98; 95\% CI, 0.96-1.00). Likewise, when $\mathrm{DLCO} \%$ (as a continuous variable) was considered in a multivariable model without $\mathrm{FEV}_{1} \%$, it was associated with respiratory grade $3+\mathrm{AE}$ at days 0 to $30(P=.02$; OR, 0.97; 95\% CI, 0.95-1.00) and days 0-90 $(P=.04$; OR, 0.98; 95\% CI, 0.96-1.00).

Table 3 shows the multivariable model results when $\mathrm{FEV}_{1} \%$ and DLCO $\%$ were considered together as continuous variables. For days 0 to 30 and 0 to 90 , only $\mathrm{FEV}_{1} \%$ was significantly associated with any grade $3+$ AE. DLCO $\%$ was significantly associated with respiratory grade $3+\mathrm{AE}$ for days 0 to 30 and 0 to 90 .

In the case of any grade $3+\mathrm{AE}$, in addition to the risk factors already considered, surgery extent and type were also explored. Surgery extent was significantly associated with any grade $3+\mathrm{AE}$, with events occurring in patients undergoing segmentectomy compared with wedge resection at days 0 to 30 (segment $=40.3 \%$, wedge $=22.7 \%$ ) and days 0 to 90 (segment $=41.8 \%$, wedge $=29.7 \%)$.

The median $\mathrm{FEV}_{1} \%$ for all patients was $50 \%$ (compared with $55 \%$ for any grade $3+$ and $53 \%$ for any respiratory grade $3+$ using the outcome-oriented approaches), and the median DLCO \% for all patients was $46 \%$ (compared with $42 \%$ and $44 \%$ for any grade $3+\mathrm{AE}$ and grade $3+$ respiratory $\mathrm{AE}$ ). Thus, we report the results using the median 
TABLE 3. Results from multivariable logistic regression models using forced expiratory volume in 1 second and carbon monoxide diffusion in the lung as continuous variables

\begin{tabular}{|c|c|c|c|}
\hline Model outcome & Predictors & Odds ratio $(95 \% \mathrm{CI})$ & $P$ value \\
\hline \multirow[t]{7}{*}{ Any grade $3+$ AE day $0-30$} & Upper lobe: yes vs no & $0.68(0.35-1.30)$ & .24 \\
\hline & Age (continuous) & $1.03(0.99-1.07)$ & .18 \\
\hline & Baseline DLCO \% (continuous) & $0.98(0.96-1.00)$ & .09 \\
\hline & Baseline $\mathrm{FEV}_{1} \%$ (continuous) & $0.98(0.96-1.00)$ & .03 \\
\hline & PS: 2 vs $0-1$ & $0.90(0.42-1.92)$ & 0.78 \\
\hline & Surgery type: thoracotomy vs VATS & $1.03(0.53-2.00)$ & 0.94 \\
\hline & Surgery extent: wedge vs segmentectomy & $0.39(0.20-0.75)$ & $<.01$ \\
\hline \multirow[t]{7}{*}{ Any grade $3+$ AE day $0-90$} & Upper lobe: yes vs no & $0.75(0.41-1.39)$ & 0.37 \\
\hline & Age (continuous) & $1.01(0.97-1.04)$ & 0.72 \\
\hline & Baseline DLCO \% (continuous) & $0.99(0.97-1.01)$ & 0.25 \\
\hline & Baseline $\mathrm{FEV}_{1} \%$ (continuous) & $0.98(0.97-1.00)$ & 0.05 \\
\hline & PS: 2 vs $0-1$ & $0.98(0.48-1.99)$ & 0.96 \\
\hline & Surgery type: thoracotomy vs VATS & $0.87(0.46-1.64)$ & 0.67 \\
\hline & Surgery extent: wedge vs segmentectomy & $0.51(0.27-0.96)$ & 0.04 \\
\hline \multirow[t]{5}{*}{ Grade $3+$ respiratory AE day $0-30^{*}$} & Upper lobe: yes vs no & $0.58(0.27-1.23)$ & 0.16 \\
\hline & Age (continuous) & $1.01(0.97-1.06)$ & 0.52 \\
\hline & Baseline DLCO\% (continuous) & $0.97(0.95-1.00)$ & 0.03 \\
\hline & Baseline $\mathrm{FEV}_{1} \%$ (continuous) & $0.98(0.96-1.00)$ & 0.07 \\
\hline & PS: 2 vs $0-1$ & $1.01(0.42-2.47)$ & 0.98 \\
\hline \multirow[t]{5}{*}{ Grade $3+$ respiratory AE day $0-90^{*}$} & Upper lobe: yes vs no & $0.59(0.29-1.18)$ & 0.14 \\
\hline & Age (continuous) & $1.00(0.96-1.04)$ & 0.97 \\
\hline & Baseline DLCO\% (continuous) & $0.98(0.96-1.00)$ & 0.05 \\
\hline & Baseline $\mathrm{FEV}_{1} \%$ (continuous) & $0.98(0.96-1.00)$ & 0.06 \\
\hline & PS: 2 vs $0-1$ & $1.15(0.52-2.53)$ & 0.74 \\
\hline
\end{tabular}

VATS, Video-assisted thoracic surgery; $P S$, performance status. *Surgery type/extent not considered because of limited number of events.

values as potential cutpoints for defining high- and low-risk categories based on baseline $\mathrm{FEV}_{1} \%$ and $\mathrm{DLCO}_{\%}$ (Table 4). An FEV 1 of less than $50 \%$ was not associated with an increased risk of grade $3+$ respiratory or any AE. However, DLCO less than $46 \%$ was significantly associated with an increased risk of respiratory and any grade $3+\mathrm{AE}$ for days 0 to 30 and 0 to 90 (Table 4). Of note, the results were similar when using the other potential cutpoints for DLCO $\%$ and $\mathrm{FEV}_{1} \%$.

\section{DISCUSSION}

Pulmonary resection is generally considered the gold standard for patients with early-stage NSCLC. For patients with impaired pulmonary function, the risks of surgical resection are a significant concern. With the increasing availability of nonoperative approaches, it is imperative that physicians have a good understanding of the relative risks of the various therapies available. The CTC classification is a useful tool that can be used to compare procedure therapy-related complications. This is the standard approach used in most oncologic studies, but it is rarely used in surgical studies. The availability of a grading system is also helpful, because many complications commonly reported in surgical series could be considered minor and less than the usual grade $3+$ reported. One example is atrial fibrillation, which was reported as $9.3 \%$ in one series of

TABLE 4. Results from the logistic regression models using median forced expiratory volume in 1 second and carbon monoxide diffusion in the lung as cutpoints

\begin{tabular}{|c|c|c|c|c|}
\hline Model outcome & Predictors & Incidence of $\mathrm{AE}(\%)$ & OR $(95 \%$ CI $)$ & $P$ value \\
\hline Grade $3+$ respiratory AE day $0-30$ & $\mathrm{DLCO} \%:<46$ vs $\geq 46$ & $11.3 \%$ vs $5.4 \%$ & $2.41(1.14-5.09)$ & .02 \\
\hline Grade $3+$ respiratory AE day $0-90$ & $\mathrm{DLCO} \%:<46$ vs $\geq 46$ & $14 \%$ vs $7.2 \%$ & $2.34(1.19-4.59)$ & .01 \\
\hline Any grade $3+\mathrm{AE}$ day $0-30$ & $\mathrm{DLCO} \%:<46$ vs $\geq 46$ & $17.1 \%$ vs $10.4 \%$ & $2.01(1.09-3.68)$ & .02 \\
\hline Any grade $3+\mathrm{AE}$ day $0-90$ & $\mathrm{DLCO} \%:<46$ vs $\geq 46$ & $19.4 \%$ vs $13.1 \%$ & $1.82(1.03-3.24)$ & .04 \\
\hline Grade $3+$ respiratory AE day $0-30$ & $\mathrm{FEV}_{1} \%:<50$ vs $\geq 50$ & $9.5 \%$ vs $7.7 \%$ & $1.29(0.64-2.61)$ & .48 \\
\hline Grade $3+$ respiratory AE day $0-90$ & $\mathrm{FEV}_{1} \%:<50$ vs $\geq 50$ & $12.6 \%$ vs $9.5 \%$ & $1.46(0.77-2.78)$ & .24 \\
\hline Any grade $3+\mathrm{AE}$ day $0-30$ & $\mathrm{FEV}_{1} \%:<50$ vs $\geq 50$ & $15.3 \%$ vs $12.6 \%$ & $1.31(0.73-2.36)$ & .37 \\
\hline Any grade $3+$ AE day $0-90$ & $\mathrm{FEV}_{1} \%:<50$ vs $\geq 50$ & $18.5 \%$ vs $14.9 \%$ & $1.40(0.80-2.46)$ & .24 \\
\hline
\end{tabular}


SR. ${ }^{10}$ By CTC criteria, atrial fibrillation would be considered grade 3 if this was incompletely controlled by medical therapy or required a device. An example of another complication, which may be over- or underreported, is atelectasis. Grade 2 atelectasis would include the use of bronchoscopy, suctioning, or chest physiotherapy for control. Grade 3 atelectasis would require operative intervention, such as a stent or laser. In the STS General Thoracic Database, atelectasis requiring bronchoscopy is classified as a complication without any consideration given to severity.

Our series demonstrated an overall incidence of grade $3+$ complications of $27.9 \%$ during the 0 - to 30 -day period. Specifically, grade 3 complications occurred in $19.4 \%$ of patients, grade 4 complications occurred in $5.9 \%$ of patients, and grade 5 (death) occurred in $1.4 \%$ of patients. These results provide a valuable benchmark for currentday morbidity and mortality after SR in these high-risk patients. In contrast with these results, the recent Radiation Therapy and Oncology Group Phase 2 study of SBRT reported a $12.7 \%$ incidence of grade $3 \mathrm{AE}$, a $3.6 \%$ incidence of grade $4 \mathrm{AE}$, and no grade $5 \mathrm{AE} .{ }^{11}$ Morbidity was less with this nonoperative intervention, although oncologic comparisons still need to be determined. A key factor to consider is that the patient groups in the 2 studies may not be directly comparable. The best comparison between these 2 therapies would be a randomized study taking similar-risk patients, using similar definitions of outcome for the entire patient cohort. This is being done by the ACOSOG, which is partnering with the Radiation Therapy and Oncology Group in a phase III study that will compare SBRT with SR for high-risk patients, using the same eligibility criteria as Z4032. This phase III study (Z4099) will be critical to help determine appropriate patient selection for operative or nonoperative therapies.

This current report provides information on 90-day outcomes for our patients. Most surgical series and even the Society of Thoracic Surgeons Database report outcomes only to 30 days. This might lead to underreporting of poor outcomes in patients who have complications resulting in transfer to long-term care facilities and who sustain grade 5 AE outside of the hospital. Our 90-day outcomes demonstrated a $2.7 \%$ mortality at 90 days, which is acceptable considering the greater than average risk of these surgical patients.

Bryant and colleagues ${ }^{12}$ recently published $30-$ and $90-$ day outcomes from a large retrospective single institution series. Their series included 1845 patients with variable operative risk and patients treated with both lobar and SRs. Discharge mortality was $3.1 \%$. An additional $1 \%$ died after discharge, within 30 days, and an additional $2.5 \%$ died within 90 days. In patients who died before 30 days, the most common cause of mortality was a pulmonary event, with risk factors being older age, occurrence of a postoperative event, greater hospital length of stay, and pneumonectomy. In patients who died between 30 and 90 days, the most common cause of mortality was natural causes, which included cancer progression.

ACOSOG previously reported morbidity and mortality in a large randomized study comparing lymph node resection with lymph node dissection. ${ }^{13}$ In that study involving 1023 standard-risk, operable patients, toxicity grade was not reported. Complications occurred in $38 \%$ of patients, and operative mortality was $1.37 \%$. The most common complication was atrial arrhythmias (14\%). Respiratory complications occurred in $7 \%$ of the patients in that large series.

We are unable to determine risk factors for 90-day mortality, because only a few grade 5 events occurred in our series. However, we evaluated the risk factors for grade $3+\mathrm{AE}$ at both 30 and 90 days. Segmentectomy is generally considered to be preferable to wedge resection as a superior oncologic procedure. ${ }^{14}$ Segmental resections are more complex, and in our study this is reflected in the higher incidence of any grade $3+\mathrm{AE}$ at days 0 to 30 and 0 to 90 .

Although $\mathrm{FEV}_{1} \%$ was a significant predictor when considered as a continuous variable for any grade $3+\mathrm{AE}$ at both 30 and 90 days, with higher values being protective, it was not significant when considered as a categoric variable. DLCO less than $46 \%$ was predictive of any grade $3+\mathrm{AE}$ and respiratory grade $3+\mathrm{AE}$ at both 30 and 90 days, suggesting that this might be a group who may have better outcomes with nonoperative therapies. Clearly, the $95 \% \mathrm{CI}$ for these OR estimates include 1.00, indicating that these results are suggestive but not definitive. This will be investigated further in Z4099.

DLCO has been demonstrated in other studies to be an independent predictor of mortality even with otherwise normal spirometry. Ferguson and colleagues ${ }^{15}$ reported results from an institutional database with 1046 patients. Within this series, there were 558 patients without chronic obstructive pulmonary disease. Postoperative predicted DLCO was the single strongest predictor of pulmonary morbidity and operative mortality in patients with and without chronic obstructive pulmonary disease in their study.

\section{CONCLUSIONS}

This randomized, prospective study demonstrates that there is no increase in morbidity or mortality with the addition of intraoperative brachytherapy to SR. In a cohort of high-risk patients, SR (with or without brachytherapy) can be undertaken with low mortality and acceptable morbidity. Segmental resection was associated with more "any" grade $3+$ AEs compared with wedge resection. Low pretreatment DLCO $\%(<46)$ was an adverse predictor of respiratory and "any" grade $3+$ AEs.

The authors thank the ACOSOG staff, in particular the leadership of Heidi Nelson and David Ota, for assistance in the development of this manuscript; all of the investigators and their site 
research teams; and the brave patients with NSCLC and their caregivers who participated in this study.

\section{References}

1. Ginsberg RJ, Rubenstein LV. Randomized trial of lobectomy versus limited resection for T1NO Non-small cell lung cancer. Ann Thorac Surg. 1995;60:615-23.

2. Fernando HC, Santos RS, Benfield JR, et al. Lobar and sublobar resection with and without brachytherapy for small stage Ia non-small cell lung cancer. $J$ Thorac Cardiovasc Surg. 2005;129:261-7.

3. Voynow G, Heron DE, Lin CJ, et al. Intraopeative (125)I Vicryl mesh brachytherapy after sublobar resection for high-risk stage I non-small cell lung cancer. Brachytherapy. 2005;4:278-85.

4. Timmerman RD, Park C, Kavanagh BD. The North American experience with stereotactic body radiation therapy in non-small cell lung cancer. J Thorac Oncol. 2007;2(Suppl 3):S101-12.

5. Dupuy DE, DiPetrillo T, Gandhi S, et al. Radiofrequency ablation followed by conventional radiotherapy for medically inoperable stage I non-small cell lung cancer. Chest. 2006;129:738-45.

6. Lee W, Daly BDT, DiPetrillo TA, et al. Limited resection for non-small cell lung cancer: observed local control with implantation of I-125 brachytherapy seeds. Ann Thorac Surg. 2003;75:237-43.

7. d'Amato TA, Galloway M, Szydlowski G, et al. Intraoperative brachytherapy following thoracoscopic wedge resection of stage I lung cancer. Chest. 1998;114: 1112-5.

8. Common Terminology Criteria for Adverse Events (CTCAE) Version 3. Washington, DC: US Department of Health and Human Services, National Institutes of Health, National Cancer Institute; 2003.

9. Williams BJ, Mandrekar JN, Mandrekar SJ, Cha SS, Furth AF. Finding optimal cutpoints for continuous covariates with binary and time-to-event outcomes. Technical Report, Division of Biostatistics, Mayo Clinic. June 2006;79.

10. Shuchert MJ, Pettiford BL, Keeley S, et al. Anatomic segmentectomy in the treatment of stage I non-small cell lung cancer. Ann Thorac Surg. 2007;84:926-33.

11. Timmerman R, Paulus R, Galvin J, et al. Sterotactic body radiation therapy for inoperable early stage lung cancer. JAMA. 2010;303:1070-6.

12. Bryant AS, Rudemiller K, Cerfolio RJ. The 30- versus 90-day operative mortality after pulmonary resection. Ann Thorac Surg. 2010;89:1717-23.

13. Allen MS, Darling GE, Pechet TTV, et al. Morbidity and mortality of major pulmonary resections in patients with early-stage lung cancer: Initial results of the randomized prospective ACOSOG Z0030 Trial. Ann Thorac Surg. 2006;81: 1013-20.

14. El-Sherif A, Fernando HC, Santos R, et al. Margin and local recurrence after sublobar resection of non-small cell lung cancer. Ann Surg Oncol. 2007;14:2400-5.

15. Ferguson MK, Vigneswaren WT. Diffusing capacity predicts morbidity after lung resection in patients without obstructive lung disease. Ann Thorac Surg. 2008;85:1158-64

\section{Discussion}

Dr Erino Rendina (Rome, Italy). Your results are remarkable, but the remarkability in terms of AEs also depends on the selection of your patients. In fact, I think when the patients have an $\mathrm{FEV}_{1}$ of approximately $50 \%$ and no other exclusion criteria, lobectomy could still be done, and this remains the gold standard because it's also preserving the quality of life. Nonetheless, looking specifically at the data you presented here, I agree with your conclusion that SR can be undertaken safely in high-risk patients with NSCLC with acceptable 30- and 90-day mortality and morbidity.

In your article, you stated that SR included wedge resection or segmental resection and could be performed by video-assisted thoracic surgery (VATS) or thoracotomy. Potential benefits of thoracoscopic resections have been reported that include equivalent oncologic outcomes to those of open surgery and less morbidity. Do you know what percentage of resections were VATS or open in your group, and did you look at whether it could have an impact on the results?
Dr Fernando. I'm going to take your first point addressing the $50 \% \mathrm{FEV}_{1}$. Our criteria were broad. They included patients who may have had restrictive disease and cardiac disease. One issue to point out is that as a surgeon you may see a patient who has an $\mathrm{FEV}_{1}$ of $45 \%$ in whom you would definitely perform a lobectomy, but you may also see a patient with an $\mathrm{FEV}_{1}$ of $55 \%$ who clearly would not tolerate a lobectomy because of other things. So our criteria were broad, but would still allow the thoracic surgeon to look at each patient and make an assessment that SR was the optimal approach for a specific patient, and allow enrollment in the study.

The second question was with the VATS versus thoracotomy. Some $65 \%$ of these patients in both groups actually had a thoracoscopic resection. It was interesting that most of the resections performed were thoracoscopic. I don't know the breakdown in terms of wedge and segment, and whether more segments were done with one approach or the other. That is planned for another analysis. Last year we presented the preliminary data on 150 patients, looking at 30- and 90-day impact on pulmonary function test, and we saw no difference between the VATS and open groups in that particular analysis.

Dr Rendina. You stated that 2 methods of brachytherapy were allowed in your study. Was your choice to use one method or the other dictated by any specific reason?

Dr Fernando. This was really at the discretion of the surgeon. We reviewed the techniques that were out there at the time the study was developed, and there was no data to support one over the other. So we left the decision to the surgeon. We have not analyzed the impact of the brachytherapy approach specifically on complications. There has been a brachytherapy quality control analysis that was presented at ASTRO by one of the radiation oncologists on the protocol, and this looked more at the ability to deliver the dose that was planned, and, again, I don't know the differences between the 2 groups.

Dr Rendina. Are there particular precautions that have to be taken with manipulating this material?

Dr Fernando. I wear lead when I do these procedures. When I'm actually sewing these in place, I don't tie down directly onto the lung. I tie extracorporeally when placing these by VATS or take a clip applier and use the clips as my knot rather than trying to tie with my hand directly onto the mesh.

Dr Rendina. In your report, the 90-day outcomes demonstrated a $2.7 \%$ mortality, which is absolutely acceptable considering the greater than average risk of these patients. However, I noticed that 1 death in the SR group included cancer progression, which is pretty surprising for patients with stage I disease. Do you have an explanation for this? Do you want to comment on this issue?

Dr Fernando. I don't know the results of that. As I said, we're planning on presenting the survival and recurrence data when this matures. I don't know whether the patient truly had a recurrence or occult metastatic disease that we didn't know about at the time, and the patient presented a month or so after resection with this. That needs to be looked at a bit more carefully.

Dr Rendina. Thank you.

Dr Thomas Waddell (Toronto, Ontario, Canada). I want to follow up on one point that Dr Rendina made and ask you to talk a bit about the new study that is going to compare this type of treatment with SBRT. This morning we heard Dr Puri talk about an analysis, 
which was retrospective, but it seems that the groups treated with SBRT and surgery were very different. Overall, $7 \%$ mortality in the surgery group and zero in the SBRT group seems to favor SBRT. So I think that the subject of who should enter these trials is critical. I take your point that you need to have an accruing trial, but I think defining what we mean by high risk is very important. How can we use this information to think about the next trial, that is, to say how do we define a high-risk person? I would say a 30day mortality of 1.4 is not that different than what Dr Allen reported based on Z0030 for lobectomy. So I would come back to Dr Rendina's point, that I'm not so sure these patients are really as high risk as you would have us believe, and certainly they are not as high risk as the patients who have been up to now dealt with by SBRT.

Dr Fernando. Actually, I would say that some of those patients treated with SBRT are probably good-risk patients as well, who are getting to the radiation oncologists without the benefit of seeing a surgeon and in some cases may have refused surgery. Every patient in this study was seen by an ACOSOG-approved thoracic surgeon, which meant they were board-certified in cardiothoracic surgery, with $50 \%$ of their practice devoted to thoracic surgery, or members of the General Thoracic Club, and they had to pass a credentialing test to be involved in this. Every patient had to be seen by a thoracic surgeon who decided if the patient was high risk for lobectomy. We can all say that we operate on patients with an $\mathrm{FEV}_{1}$ of $40 \%$ or $35 \%$ and sometimes perform lobectomies on those patients. Every patient has a specific set of comorbidities that allows you as a surgeon to make that judgment. If we had made this trial restrictive and only taken $30 \%$ or less or $40 \%$ or less, or whatever number we would have chosen, it would have taken twice as long to accrue to this study. This next study is going to be looking at patients with exactly the same set of inclusion criteria. Every patient will have to be seen by a thoracic surgeon. The only difference is that the tissue diagnosis will have to be made up front so we avoid the problem that other SBRT studies have made by including patients who don't have a tissue diagnosis. We are going to have common definitions of outcome, including complications and recurrence, because there are also differences in recurrence rates between the SBRT and surgical studies that can be explained in part by differences of definition. So this next study will give us our best chance to see what the role of surgery is for this group of patients.

Dr Todd Demmy (Buffalo, $N Y$ ). Do you have any data on the discharge independence of these patients? I think that's the other element that we have to capture when we start thinking about comparative studies. It was already stated this morning in the lecture that if it's less invasive, it tends to win, so SBRT already has an edge on that. Are you going to collect data to show that these patients don't go to nursing homes and end up with a lot of repeat visits beyond the 90-day mortality end point? Are you going to look at these functional and quality of life outcomes?

Dr Fernando. With Z4032, we didn't include that as an end point, but the Z4099 study, which opened last week, the case report forms specifically ask those questions about where the patient goes, home or to an acute care facility. I think that is an important outcome to measure.

Dr Scott Swanson (Boston, Mass). That was an outstanding presentation, and you are to be commended for doing this trial because it's critical for us as surgeons. The outcome data are amazing, really good mortality and morbidity.

Do you have any data on lymph node sampling? I know that's not the point of this study, but as we go forward comparing with SBRT, certainly that is a major difference. Was that part of this study? Was there any requirement? Do you have any details on that?

Dr Fernando. Lymph node counts were not collected in our case report forms. We are planning an analysis comparing VATS and thoracotomy, which Michael Kent from Beth Israel Deaconess is going to lead. We are going to be looking at all the operative and path notes that are available as part of the source data, even though that specific information was not collected in the case report forms up front. We hope to have that information within the next 6 months to present to everybody when we look at the differences for the patients who had VATS or thoracotomy.

Dr Servet Bolukbas (Wiesbaden, Germany). During the evaluation of your patients, there is a high possibility of detecting chronic obstructive pulmonary disease. Were there any attempts to treat those patients with drugs, to send them to pulmonary rehabilitation to improve $\mathrm{FEV}_{1}$, to switch the patients from high to normal risk?

Dr Fernando. Not specifically in this group. I think the difference between these patients and patients in the National Emphysema Treatment Trial study, for example, is that you don't really have the luxury of 6 weeks to perform pulmonary rehabilitation in these patients. You want to try and deal with the cancer within a reasonable time frame. So we have not been putting patients through pulmonary rehabilitation before resecting lung cancers.

Dr Bolukbas. But in stage I, I think there is time to do pulmonary rehabilitation for 6 weeks.

\section{Dr Fernando. Perhaps.}

Dr Joel Cooper (Philadelphia, Pa). Just for point of emphasis, I want to recapitulate. I am concerned with people labeling an $\mathrm{FEV}_{1}$ of $50 \%$ as defining "high risk" because it's not, and the pulmonary physicians and radiotherapists are going to use that definition to indicate that patients are high risk when they are not, and that will turn influence selection criteria for these trials. Remember, chronic obstructive pulmonary disease is a couple of pulmonary diseases-airways disease and emphysema, 2 very different conditions, both with very different risk factors. The $\mathrm{FEV}_{1}$ can be $25 \%$ with no emphysema because it's due to small airways disease, or it can be due to loss of elastic recoil, namely, emphysema. Diffusing capacity is a little different. A person with an $\mathrm{FEV}_{1}$ of $25 \%$ whose computed tomography scan doesn't show much emphysema and whose diffusing capacity is $35 \%$ to $40 \%$ is not a particularly high-risk patient for a lobectomy if you choose well. In summary, $\mathrm{FEV}_{1}$ alone does not adequately define risk for resection and potentially does a great disservice to patients. Furthermore, it ignores the importance of the surgeon's judgment and experience in selecting appropriate patients for surgical resection.

Dr Fernando. I will say that the American College of Chest Physicians in their guidelines used $80 \%$ for their cutoff for what defined high or low risk, and they also recommended more invasive evaluation of those patients, including split-lung ventilation/ perfusion scans, which seems kind of ridiculous for patients with such a high FEV\%. That's something that Frank Detterbeck is investigating in another study. I also think there is a real problem with trying to accrue patients to studies, and if we only used 
a low $\mathrm{FEV}_{1}$ cutoff point, we would have taken forever to do this, and perhaps not completed the study.

Dr Joachim Schirren (Wiesbaden, Germany). I have 2 questions. First, you had a consensus conference about the surgical procedure from 9 centers? Second, why do you use DLCO? Currently, I think it's important to see the dynamics of the patient during spiroergometry. The last point is, if you have a patient with a DLCO that you described, you walk with him, 3 stairs, 4 stairs, and then you decide if you will operate. It would be interesting to see how the DLCO was, how the physiologic status was of the patient to steps, stairs, and, second, to see how the spiroergometry was. Can you say something about this?

Dr Fernando. We didn't use a stair-climbing test as such. Actually, I think it's 35 sites that participated. The sites listed were the top 5 accruing sites, plus some of the radiation staff who were involved in the study design and implementation. The way the trial developed is that we had a 2-day conference where we got together and reviewed what the best literature out there was. We invited some pulmonologists and radiation oncologists to the meeting as well. Then we broke off into groups to work out the optimal criteria that would define a high-risk group, as well as the optimal study for these patients. So this was worked out some time ago from that consensus conference. In fact, we used the same criteria for $\mathrm{Z} 4033$, which is the radiofrequency ablation study that also recently completed accrual. The only difference there was that the surgeon had to see the patient and state that he or she didn't think the patient was even a candidate for sublobar resection. As you can imagine, it took a lot longer to accrue patients to that 50-patient trial because many of these patients were probably treated with resection. 